\title{
DEVELOPMENT AND IMPROVEMENT OF ASSESSMENT MEANS OF TECHNICAL SYSTEMS QUALITY IN THE PROCESS OF MAINTENANCE
}

\author{
K.Z. Bilyatdinov ${ }^{1, a}$, E.A. Krivchun ${ }^{2}$ \\ ${ }^{1}$ ITMO University, Kronverkskiy prospekt, 49-A, St. Petersburg, 197101, Russia \\ ${ }^{2}$ Saint-Petersburg State University of Aerospace Instrumentation, 67, Bolshaya Morskaia \\ str., Saint-Petersburg, 190000, RUSSIA \\ E-mail: ${ }^{a}$ k74b@mail.ru
}

The paper proposes rational instruments for innovative development and improvement of methods of technical systems' quality assessment in the process of maintenance. The proposed innovations are based on the application of a number of interconnected model, methods and computer programs for their implementation, which together allow to decrease time and resources spent on assessment of the quality of systems and (or) elements (products) of the systems. It is recommended to apply the proposed methods in order to make timely managerial decisions based on the results of technical systems' quality assessment in the process of maintenance.

Keywords: method, system, assessment, rating, quality, solution, methodology, model, principles 


\section{Purpose of the study}

The topicality of establishment of innovative ways of development and improvement of assessment means of technical systems (hereinafter, systems) quality is proved by contemporary requirements for improvement of management and decision making process. The purpose of this trend is increase of effectiveness of systems functioning which in its turn leads to the necessity of development of new scientifically justified methodological, technical and technological decisions [1, $2,7,11]$.

Nowadays one of the rational ways of developing such decisions can be creation of new and improvement of existing methods of quality assessment, rational procedures of information processing and models of systems management in the process of exploitation. Creation and introduction of such decisions considerably influences development of production resources, which is of primary importance considering the fact that effective and stable maintenance of systems has great value for economics $[7,9,10]$. The proposed innovations help to level existing contradictions between and increasing volume of heterogeneous sources of structured and unstructured data of studied systems and objective necessity to make timely managerial decisions on the basis of existing means of quality assessment $[2,8,9,11]$. Thus, the essence of the innovative approach lies in the improvement of existing means of quality assessment with consideration of the specificity of maintenance and perspective capacities of assessed systems.

In its turn, realization of the proposed innovative approach will ensure formation of a complex of scientifically justified technical, technological and methodological decisions. In practice, these decisions will form prerequisites for the increase of management effectiveness without expenditures of extra resources $[2,9]$.

Obviously, in the context of the presented problem innovative development and improvement of existing methods will consist in the increase of management effectiveness and decision making at the stage of systems maintenance. This purpose will be achieved through a considerable decrease of time and resources expenditures for the assessment of quality by means of development and implementation of a complex of innovative methodologies of quality assessment and software for their realization (hereinafter, the methodologies). In actual practice, implementation of the methodologies will lead to decrease of time needed for making justified managerial decisions in the conditions of increased volume of the information used for systems quality assessment.

The current level of development of information technologies and computerization of the society allows the authors to study the above presented problem in relation to contradictions of management of technical-organizational systems [1, p. 40]. Following this, the authors group current contradictions of management into four levels: personal, social, technical and technical-organizational. Within the proposed innovative approach the developed methodology is mainly applied at technical and technical-organizational levels [1, p. 42].

\section{Model description}

Nowadays, a variety of firmware, other technical systems and present development of technologies lead to the necessity of a complex use of information from internal and external information resources. Development of information technologies allows formation of information reserves (hereinafter, IR) of systems quality assessment. principles:

In consideration of the above-mentioned, the described innovations are based on three 
The first principle consists in ensuring effectiveness of methods and minimization of risks of making a wrong decision based on the results of quality assessment.

The second principle consists in universality, practical orientation of methods, cheapness and simplicity of their realization in different systems without considerable expenditures of time and resources for use of telecommunications, computer aids and preparation of staff.

The third principle consists in structure-functional classification of assessed systems (products) within technical systems. The essence of this principle is classification of a number of assessed systems by place (conditions) of maintenance, after which these groups of systems are divided into other groups (subgroups) according to their function and tasks [2, p. 199].

Following these three principles, the most rational approach for effective application of the methodologies is creation of a universal model of systems' support in the process of maintenance.

The proposed model presents a description of the procedure of systems' support in the process of maintenance. The purpose of the model is rational organization of the work needed for development and improvement of systems. This model is universal and applicable for various systems' support with consideration of the specificity of their functioning and possible unfavorable conditions. The model aims at identification of a role, place, content and the most rational application of IR, methodologies and software for systems quality assessment in the process of maintenance in the conditions of saving time and resources [6, p. 105].

\section{Methodology of assessment of systems effectiveness}

This methodology is developed on the basis of modified DEA method [3, p. 612]. In actual practice, the methodology is realized by a software "Analysis and assessment of systems effectiveness" (Certificate of the state registration of software № 2020610389, 14.01.2020, registered by Biliatdinov K.Z.). The methodology and software are aimed at analysis and assessment of effectiveness of systems and (or) one system in different periods of their (its) functioning.

The methodology, realized by the software, makes all necessary calculations for creation of tables, graphs and diagrams and for systematization of received values of correlation dependence of indices of systems functioning. Also, it allows to compare systems (periods of time) by the result and (or) index of systems functioning or by correlation of spent resource and the result, moreover, it can make ratings of systems.

The main positive effect from implementation of the methodology and software consists in clarity and visibility of the results, also, in decrease of time and resources spent on assessment of effectiveness and increase of validity of managerial decisions achieved through the possibility of comparison and necessary specification of spent resources in the process of analysis and assessment of a system's effectiveness [3, p. 617].

Methodology of assessment of systems stability [2]. In actual practice, the methodology is realized by a software "Assessment of systems stability" (Certificate of the state registration of software № 2020615328, 21.05.2020, registered by Biliatdinov K.Z.). Purpose of the methodology and software:

1. Assessment of stability of systems' activities of a function (functions) and (or) ensuring of stability of functioning depending on the results of influence of unfavorable conditions in a given period.

2. Calculation of a coefficient of a system's stability as a quality index in the sphere of stability ensuring.

3. Analysis of the influence of values of quality indices of systems (products) on the stability 
of a system and justification of recommendations for the increase of its stability by means of improvement of values of quality indices of systems (products) within it (improvement of maintenance, technical support and staff preparation, choice of modernization direction or justification of the necessity to create new systems).

\section{Complex methodology of systems quality assessment in the process of maintenance}

The basic sequence of actions when implementing this methodology can be of two variants depending on the kind of information used in the process of assessment: information based on statistical data or statistical and expert information. The essence of the methodology is in step-by-step application of interconnected calculation tables for quality assessment based on statistical and expert information with the possibility to identify sources of information [4, p. 21].

Method of rational processing of information resources and formation of IR of systems (hereinafter, the method) [2, 6]. In actual practice, the method is realized by a software "Realization of the method of rational processing of information resources and formation of information reserves of a system” (Certificate of the state registration of software № 2020610335, 13.01.2020, registered by Biliatdinov K.Z.).

The method is aimed at considerable decrease of man-hours and time for collection and processing of information, also, at formation and renewal (upkeep in up-to-date condition) of IR of quality assessment in conditions of increased volumes of processed information.

The essence of the method: information and applied software tools are systematized in IR, which consist of a database with main data about a system and of an archive. Routine tasks of information collecting and processing are sequentially completed on two stages [6, p. 108].

The first stage - work with IR of a system. The task is completed by addressing IR. During this, the request first comes to the database, then to the archive. In the majority of cases, officials (decision-makers) are satisfied with the response from the database. In this case, the work finishes with the closure of the first stage. As the result of this, IR of a system are replenished with the information of a completed task.

The second stage - addressing external information resources. If IR of a system do not contain the required information in corpore, then, an official (decision-maker) may decide to search the information on the Internet and (or) send a request to other organizations and institutions [6, p. 110].

\section{Conclusion}

To conclude, it is necessary to emphasize that the proposed innovative approaches help to increase universality of the methodology. Thus, the methodology and its elements can be effectively applied to three main spheres:

1. Increase of effectiveness of management, maintenance and technical support of assessed systems.

2. Increase of quality of research works and development projects in the area of creation and modernization of systems.

3. Increase of quality of staff preparation in the sphere of maintenance and technical support of hardware-in-the-loop tools and technical systems.

The most effective direction of application of the proposed methods (methodologies and software) is their application as key part of integrative reserves of increase of effective functioning of systems in the process of maintenance. 


\section{References}

[1] Biliatdinov K.Z. Contradictions of the process of management in the contemporary world // Age of Quality, issue 3, 2014, p 40-43 (in Russian).

[2] Biliatdinov K.Z., Meniailo V.V. Methodology of systems quality assessment in the sphere of big technical objects // Age of Quality, issue 2, 2020,p. 198-214 (in Russian).

[3] Biliatdinov K.Z., Meniailo V.V. Modified DEA method and methodology of effectiveness assessment of technical systems // Information technologies, issue 11, 2020, p. 611-617 (in Russian).

[4] Biliatdinov K.Z., Complex methodology of technical systems quality assessment in the process of maintenance // Scientific and technical bulletin of Volga region, issue 11, 2020, p. 20-23 (in Russian).

[5] Biliatdinov K.Z. Methodology of assessment of probabilistic characteristics of technical systems in the process of maintenance // Scientific and technical bulletin of Volga region, issue 12, 2020, p. 21-24 (in Russian).

[6] Biliatdinov K.Z., Shestakov A.V. Formation and use of information reserves in support of big technical systems // Proceedings of telecommunication universities, vol. 6, issue 4, 2020, p. 104-110 (in Russian).

[7] Duer S. Assessment of the operation process of wind power plant's equipment with the use of an artificial neural network // Energies. 2020. № 13. Art. 2437.

[8] Gerami J. An interactive procedure to improve estimate of value efficiency in DEA // Expert Systems with Applications. 2019, № 137, p. 29-45.

[9] Golabchi A., Han S., AbouRizk S. A simulation and visualization-based framework of labor efficiency and safety analysis for prevention through design and planning // Automation in Construction. 2018, vol. 96, p. 310-323.

[10] Trevino M. Cyber Physical Systems: The Coming Singularity. PRISM. 2019, 8 (3), p.2-13.

[11] Yazdi M. Introducing a heuristic approach to enhance the reliability of system safety assessment // Quality and Reliability Engineering International. 2019, vol. 35, issue 8, p. 2612-2638. 